\title{
Schrödinger Transform of Image: A New Tool for Image Analysis
}

\author{
Liantang Lou ${ }^{1}$, Hua Zeng ${ }^{1 *}$, Jipeng Xiong ${ }^{1}$, Lingling $\mathrm{Li}^{2}$ and Wenliang Gao ${ }^{1}$ \\ ${ }^{1}$ School of Science, Hubei Province Key Laboratory of Intelligent Robot, \\ Wuhan Institute of Technology, Wuhan \\ ${ }^{2}$ Department of Computer Science and Application, ZhengZhou Institute of \\ Aeronautical Industry Management, Zhengzhou \\ China
}

\section{Introduction}

Image segmentation is the process of separating or grouping an image into different parts . These parts normally correspond to something that human beings can easily separate and view as individual objects. Computers have no means of intelligently for recognizing objects, and a large number of different methods have been developed in order to segment images, ranging from the simple thresholding method to advanced graph-cut methods. The segmentation process is based on various features found in the image. Those features might be histograms information, information about the pixels that indicate edges or boundaries or texture information and so on.

Approaches of Image processing and analysis based on partial differential equation, such as deformable models or snakes (Terzopoulos et al., 1987; Kass, et al., 1987), balloon models (Cohen, L. D., 1991; Cohen, L. D. \& Cohen, I., 1993), geometric models (Caselles et al., 1993), discrete dynamic contour models (Lobergt \& Viegever, 1995), geodetic active contours (Caselles et al., 1995) and topology adaptive deformable model (McInerney \& Terzopoulos, 1999), whose physical background is principle of minimum action or force equilibrium in classical mechanics, are being extensively applied to image segmentation, image smooth, image inpainting, extraction of boundary and so on. $\mathrm{Xu}$ and Prince analyzed the reason why snake methods have poor convergence to boundaries with large curvatures and replaced the gradient field with the gradient vector field (GVF), which has a larger capture region and slowly changes away from the boundaries (Xu \& Prince, 1998). Consequently, the dependence on initial positions is decreased but the field can attract the moving contour to the right position. Parametric deformable models have high computational efficiency and can easily incorporate a priori knowledge. However, these models cannot naturally handle topological changes and are sensitive to initial conditions. Geometric deformable models are based on the level set method (Osher \& Sethian, 1988), which was initially proposed to handle topological changes during the curve evolution. Geometric deformable models have the advantage of naturally handling the topological

${ }^{*}$ Corresponding Author 
changes and are widely studied for medical segmentation (Malladi \& Sethian, 1996). Another popular geometric model is proposed by Chan and Vese (Chan \& Vese, 1999; 2002). Chan-Vese's model is a simplified version of the Mumford-Shah energy model. The algorithm extracts the desired object through simultaneously minimizing the intensity variations inside and outside the contour. In 1997, Cohen and Kimmel described a method for integrating object boundaries by searching the path of a minimal active deformable model's energy between two points (Cohen, L. D. \& Kimmel, 1997). But they are easy to fall into the local minimum, sensitive to noise, do not have topology adaptive, poor convergence to concave boundaries. Lou and Ding used point tracking by estimating the maximum probability of a particle in quantum mechanics moving from one point to another, and did not impose any smoothness constraints to ensure the extraction of the details of a concave contour (Lou \& Ding, 2007a). To overcome the main drawbacks of global minimal for active contour models that the contour was only extracted partially for low SNR images, maximal probability method of boundary extraction based on particle motion was proposed (Lou et al, 2007b). Schrödinger transform of image was first given by Lou, Zhan, Fu and Ding, and the probability $P(b, a)$ that a particle moved from a point a to another point was computed according to I-Type Schrödinger transform of image (Lou et al, 2008). In the chapter, a new tool for image, Schrödinger Transform of image, is investigated.

The reminder of the chapter was organized as follows. First, we gave physical explanation of boundary extraction from the point view of classical mechanics and quantum mechanics in Section 2. Next, we defined Schrödinger transform of image, discussed its properties and computation in Section 3. Then we investigated scale parameter and potential function of Schrödinger transform in Section 4. And then, we constructed high and low pass filter and carried through automatic contour extraction for multiple objects using I-type Schrödinger transform of image, segmented image using deformation of II-type Schrödinger transform of image in Section 5. Finally, we gave our conclusion in Section 6.

\section{Physical explanation of boundary extraction}

Boundary extraction is belong to field of image progressing while classical mechanics and quntumn mechanics are pure physical concept. However, we can find their common ground from the point of view of particle motion. Boundary of object can be thought of as trajectory of moving particle while the law of motion of particle were investigated in classical mechanics and quntumn mechanics using two determinancy and nondeterminancy method.

\subsection{Physical explanation of boundary extraction from the point of view of classical mechanics}

Deformable models are the elastic curves defined within an image domain that can move under the influence of internal forces arising from curve smoothness and external forces computed from the image data. The internal and external forces are so defined that the deformable contour has a minimum energy at the true object boundary. The following mapping can represent the deformable contour model:

$$
\begin{aligned}
\mathbf{x}(s): & {[0,1] \rightarrow R^{2} } \\
& s \mapsto(x(s), y(s))
\end{aligned}
$$


where $s \in[0,1]$ is the parameterization variable of the object boundary and 0 and 1 correspond to the start and end points of the boundary. The deformable contour is a curve $\mathbf{x}(s)=(x(s), y(s))$ that moves in the spatial domain of the image to minimize the energy:

$$
E=\int_{0}^{1} \frac{1}{2}\left(w_{1}\left|\mathbf{x}^{\prime}(s)\right|^{2}+w_{2}\left|\mathbf{x}^{\prime \prime}(s)\right|^{2}\right)+E_{e x t}(\mathbf{x}(s)) d s
$$

where $w_{1}, w_{2}$ are the weighting parameters controlling the contour's tension and rigidity respectively. $\mathbf{x}^{\prime}(s)$ and $\mathbf{x}^{\prime \prime}(s)$ are the first and second order derivatives of $\mathbf{x}(s)$ with respect to $s$. The external energy $E_{\text {ext }}$ is a function of the image data depending on the goal of application. For object boundary extraction, it is defined as the image gradient. Suppose $\mathbf{x}(s)$ has a local minimum of $E$ at $s$, the following Euler-Lagrange Equation has to be satisfied:

$$
w_{1} \mathbf{x}^{\prime \prime}(s)-w_{2} \mathbf{x}^{\prime \prime \prime \prime}(s)-\nabla E_{e x t}=0
$$

To solve Eq. (3), the boundary conditions of $\mathbf{x}(s)$ should be given. If the boundary is closed, a periodic boundary condition $\mathbf{x}(0)=\mathbf{x}(1)$ can be attached. Each term in Eq. (3) is considered as a force applied on the curve. The internal force $w_{1} \mathbf{x}^{\prime \prime}(s)-w_{2} \mathbf{x}^{\prime \prime \prime \prime}(s)$ resists stretching and bending while the external potential force $-\nabla E_{\text {ext }}$ pulls the contour towards the boundary. Thus, the object boundary is obtained either when the force equilibrium of Eq. (3) or a minimum of the energy in Eq. (2) is reached.

\subsection{Physical explanation of boundary extraction from the point of view of quantum mechanics}

The deformable contour $\mathbf{x}(s)=(x(s), y(s))$ can further be considered as the path of a moving particle in the image if the parameterization variable $s$ is replaced by the time variable $t$. By referring the work of Feynman and Hibbs (Feynman \& Hibbs, 1965), we explain boundary extraction from the point of view of quantum mechanics as follows:

Suppose a particle moves from the position $a$ at the time $t_{a}$ to the position $b$ at the time $t_{b}$, e.g., $a=\mathbf{x}\left(t_{a}\right), b=\mathbf{x}\left(t_{b}\right)$. According to the theory of quantum mechanics, the amplitude, $K(b, a)$, called kernel or propagator, contains the total contribution of all paths between $a$ and $b$, which is different from Eq. (2) where only a specific path from $a$ to $b$ with the minimum energy is concerned. In order to distinguish these two types of contours, we refer to the contour determined by $K(b, a)$ as the quantum contour, denoted as $Q(t)$, while the traditional deformable contour determined by minimizing the energy between $a$ and $b$ as the classical contour, denoted by $X(t)$. Obviously, the classical contour $X(t)$ is considered as a specific case of the quantum contour $Q(t)$ when a single path is concerned. In physics, the energy functional of a path, $E$, is defined by:

$$
E=\int_{t_{a}}^{t_{b}} L\left(\mathbf{x}^{\prime}(t), \mathbf{x}(t), t\right) d t
$$

where $L$ is the Lagrangian function of system. For a moving particle with the mass $m$, and potential $V(\mathbf{x}, t)$, the Lagrangian function is determined by:

$$
L=\frac{m}{2}\left(\mathbf{x}^{\prime}(t)\right)^{2}-V(\mathbf{x}, t)
$$


Comparing Eq. (4) to Eq. (2), we find that the Lagrangian functional of a path in Eq. (4) is similar to the energy functional of a contour curve in Eq. (2). Thus, we refer to deformable model as the motion of a particle described by classical mechanics.

In quantum mechanics, the total contribution of all paths between $a$ to $b$ is calculated by

$$
K(b, a)=\sum_{R(a, b)} \phi(\mathbf{x}(t))
$$

where $R(a, b)$ is the set of all paths between $a$ and $b . \phi(\mathbf{x}(\mathbf{t}))$ is the contribution of a path $\mathbf{x}(t)$ with a phase proportional to its energy $E(\mathbf{x}(t))$, i.e.,

$$
\phi(\mathbf{x}(t))=C \cdot e^{(2 \pi j / h) E(\mathbf{x}(t))}
$$

where $h$ is the Planck's Constant and $C$ is a constant.

According to the theory of quantum mechanics, the probability ${ }^{1}$ of a particle moving from the position $a$ at time $t_{a}$ to the position $b$ at $t_{b}$, denoted by $P(b, a)$, is equal to the square amplitude of $K(b, a)$, i.e.,

$$
P(b, a)=|K(b, a)|^{2}
$$

For a system with a simple Lagrangian function, $K(b, a)$ can be calculated directly from the path integral ((Feynman \& Hibbs, 1965)) while for a system with a complex Lagrangian function, it is difficult and time-consuming to estimate the value of $P(b, a)$ from $K(b, a)$. In order to avoid such difficulty, we estimate the probability of a particle moving from point $a$ to point $b$ directly from specific particle models, e.g., a free particle or a particle moving through a Gaussian slit, where their motions can be used to describe the boundary of an object of interest with a known probability density of appearing at a point.

There is a stronger motivation of adapting the quantum mechanics than the similarity between equations (2) and (4). Snakes, Deformable models, level set methods, etc, are all based on classical mechanics in a form of partial differential equation. Although classical physics is adequate to explain virtually all phenomena one will ever directly experience in one's life, certain phenomena cannot be explained by classical physics. In many respects, quantum mechanics presents the physics that underlies physical reality at its most basic level. Quantum theory can be thought of as the generalization of classical mechanics and many non-classical phenomena that do not have a classical analog are known in the quantum physics world. The relationship between classical and quantum mechanics is of central importance to the philosophy of physics. Classical mechanics extends the elementary Newtonian concepts to the Lagrangian and Hamiltonian formulations, to the least action principle, to the angle-action variables, etc, in ways that are the essential framework of quantum mechanics. However, there are significant distinctions between the two theories that arise not because of quantization, but rather from the nonessential tendency to describe macroscopic systems by instantaneous values for position, speed and acceleration, and microscopic systems by time-averaged position probability densities. Probability is a bridge between classical mechanics and quantum mechanics. The detail discussion of this relationship is out of this chapter's scope, which can be founded by hundreds of literatures in quantum mechanics field.

\footnotetext{
${ }^{1}$ Here the probability refers to a relative probability.
} 


\section{Schrödinger transform of image}

For a complex system, however, it is difficult to calculate the value of $P(b, a)$. In 1995, Williams and Jacobs derived the probability that a particle with a random walk passes through a given position and orientation on a path joining two boundary fragments, which is obtained by the product of two vector-field convolutions (Williams \& Jacobs, 1995).

Although some specific particle motions have been considered, a general analytic expression of the probability for complex system is still open. In order to calculate the value of $P(b, a)$, a numerical approximation of $P(b, a)$ is needed. In this section, we will try to compute the probability $P(b, a)$ by using Schrödinger transform of image.

\subsection{Definition of Schrödinger transform of image}

The active contour model or Snake model had their profound physical background. If the parameter $s$ in the deformable contour curve $\mathbf{x}(s)=(x(s), y(s))$ could be understood as time $t$, object contour curve $\mathbf{x}(t)$ could be considered as the path of the particle in plane motion.

Suppose a particle moves from the position $a$ at the time $t_{a}$ to the position at the time $t_{b}$,e.g., $a=x\left(t_{a}\right), b=x\left(t_{b}\right)$. According to the theory of quantum mechanics, the probability of a particle moving from the position $a$ to $b$ at $t_{b}$, denoted by $P(b, a)$, is dependent on the kernel $K(b, a)$, which is the sum of all paths contribution between $\mathbf{x}_{a}$ and $\mathbf{x}_{b}$, i.e.,

$$
K(b, a)=\sum_{R(a, b)} \phi(x(t)),
$$

where $R(a, b)$ is the set of all paths between $\mathbf{x}_{a}$ and $\mathbf{x}_{b} \cdot \phi(\mathbf{x}(t))$ is the contribution of a path $\mathbf{x}(t)$ with a phase proportional to its energy $E(\mathbf{x}(t))$, i.e.,

$$
\phi(\mathbf{x}(t))=C e^{(2 \pi i / h) E(\mathbf{x}(t))}
$$

where $h$ is the Planck's Constant, $C$ is a constant, and $E=\int_{t_{a}}^{t_{b}}\left(\frac{m}{2}\left|\mathbf{x}^{\prime}(t)\right|^{2}-V(\mathbf{x}, t)\right) d t$ is the energy functional of path. The probability from point $\mathbf{x}_{a}$ at time $t_{a}$ to point $\mathbf{x}_{b}$ at time $t_{b}$ is the square of absolute value of kernel $K(b, a)$ from $\mathbf{x}_{a}$ to $\mathbf{x}_{b}$, that is,

$$
p(a, b)=|K(b, a)|^{2} .
$$

We must solve the problem of computing the kernel $K(b, a)$ to introduce law of particle motion in quantum mechanics into image processing and analysis. For a system with a simple Lagrangian function, $K(b, a)$ can be calculated directly from the path integral (see 0 ) while for a system with a complex Lagrangian function, it is difficult and time-consuming to estimate the value of $P(b, a)$ from $K(b, a)$. Replacing the kernel $K(b, a)$ with the wave function $u(\mathbf{x}, t)$ in the position $\mathbf{x}$ at the time $t$, then $u(\mathbf{x}, t)$ satisfied the following Schrödinger equation:

$$
\bar{h} i \cdot \frac{\partial u}{\partial t}=-\frac{\bar{h}^{2}}{2 m}\left(\frac{\partial^{2} u}{\partial x^{2}}+\frac{\partial^{2} u}{\partial y^{2}}\right)+V(\mathbf{x}, t) u(\mathbf{x}, t)
$$


where $\bar{h}=h / 2 \pi=1.054 \times 10^{-27} \mathrm{crg} \cdot s, \quad i$ is imaginary unit. So, the relation between the probability $P(b, a)$ and image gradient $G(\mathbf{x})$ can be given in Fig. 1 .

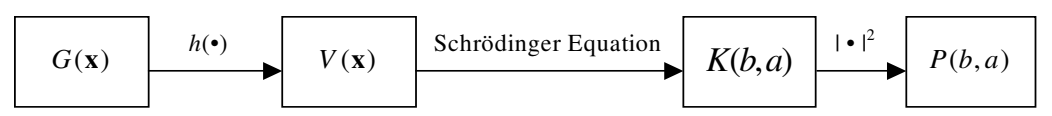

Fig. 1. the relation between the probability $P(b, a)$ and image gradient $G(\mathbf{x})$

We could rewrite Eq.(11) as the initial-value problem:

$$
\left\{\begin{array}{c}
i \cdot u_{t}+a \Delta u=v(\mathbf{x}) u \\
\left.u\right|_{t=0}=\varphi(\mathbf{x})
\end{array}\right.
$$

Continuous Schrödinger transform of image $\varphi(\mathbf{x})$ based on $v(\mathbf{x})$ is defined as the solution of Eq.(12). And the transform is called I-type Schrödinger transform when $v(\mathbf{x})=0$, otherwise the transform is called II-type Schrödinger transform.

By applying Fourier transform to equation (12) and making use of the properties of Fourier transform, we have

$$
\left\{\begin{array}{c}
i \cdot \hat{u}_{t}-a|\mathbf{y}|^{2} \hat{u}=\hat{v}(\mathbf{y}) * \hat{u} \\
\left.\hat{u}\right|_{t=0}=\hat{\varphi}(\mathbf{y})
\end{array}\right.
$$

where the mark ' $*$ ' denotes convolution of two functions, ' $\wedge$ ' denotes Fourier transform of function. When $v(\mathbf{x})=0$, both $u(\mathbf{x}, t)$ and $\hat{u}(\mathbf{y}, t)$ have the following analytic solutions (see 0):

$$
\begin{gathered}
\hat{u}=e^{-a i t|\mathbf{y}|^{2}} \hat{\varphi}(\mathbf{y}) \\
u(\mathbf{x}, t)=\frac{1}{4 \pi a i t} \iint e^{\frac{i}{4 a t}|\mathbf{x}-\mathbf{y}|^{2}} \hat{\varphi}(\mathbf{y}) d \mathbf{y}, \quad \mathbf{x} \in R^{2}, t>0,
\end{gathered}
$$

When $v(\mathbf{x}) \neq 0, \quad u(\mathbf{x}, t)$ and $\hat{u}(\mathbf{y}, t)$ also have analytic solutions (L. C. Evans, 1998), but they are too complex to be used to compute their numerical solutions. We give the following definition of discrete Schrödinger transform of image because of Eq.(12) and Eq.(13):

Supposed both $\varphi(\mathbf{x})$ and $v(\mathbf{x})$ are $m \times n$ images, then two-dimensional discrete Schrödinger transform of image $\varphi(\mathbf{x})$ based on $v(\mathbf{x})$ is expressed with the following differential equation which its Fourier transform satisfies:

$$
\left\{\begin{array}{c}
i \cdot \overrightarrow{\hat{u}}_{t}=\left(V+a|\mathbf{y}|^{2}\right) \overrightarrow{\hat{u}} \\
\left.\overrightarrow{\hat{u}}\right|_{t=0}=\overrightarrow{\hat{\varphi}}
\end{array},\right.
$$


where $\overrightarrow{\hat{u}}_{t}$ is $m n$-dimensional column vector formed by concatenating all the rows of $m \times n$ matrix $\hat{u}_{t} \cdot m n \times m n$ matrix $|\mathbf{y}|$ was diagonal matrix whose diagonal elements express distance. $m n \times m n$ matrix $V$ is a block cyclic matrix, i.e.,

$$
V=\left(\begin{array}{cccc}
V_{0} & V_{m-1} & \cdots & V_{1} \\
V_{1} & V_{0} & \cdots & V_{2} \\
\vdots & \vdots & \vdots & \vdots \\
V_{m-1} & V_{m-2} & \cdots & V_{0}
\end{array}\right),
$$

where $V_{i}$ is a cyclic matrix,

$$
V_{i}=\left(\begin{array}{cccc}
\hat{v}(i, 0) & \hat{v}(i, n-1) & \cdots & \hat{v}(i, 1) \\
\hat{v}(i, 1) & \hat{v}(i, 0) & \cdots & \hat{v}(i, 2) \\
\vdots & \vdots & \vdots & \vdots \\
\hat{v}(i, n-1) & \hat{v}(i, n-2) & \cdots & \hat{v}(i, 0)
\end{array}\right),
$$

Discrete Schrödinger transform of image $\varphi(\mathbf{x})$ based on $v(\mathbf{x})$ is denoted by $\mathbb{S}_{v}(\varphi)$ while Itype discrete Schrödinger transform of image $\varphi(\mathbf{x})$ is denoted by $\mathbb{S}(\varphi)$.

Obviously, the solution of Eq.(16) is

$$
\mathbb{S}_{V}(\varphi)=\hat{u}(\mathbf{y})=e^{-i t\left(V+a|\mathbf{y}|^{2}\right)} \hat{\varphi}(\mathbf{y})
$$

If the matrix $V+a|\mathbf{y}|^{2}$ can be diagonalized, that is, $V+a|\mathbf{y}|^{2}=P^{-1} D P$, then

$$
\hat{u}(\mathbf{y})=P^{-1} \operatorname{Diag}\left(e^{-i t d_{1}}, e^{-i t d_{2}}, \cdots, e^{-i t d_{m n}}\right) P \hat{\varphi}(\mathbf{y}),
$$

where $D=\operatorname{Diag}\left(d_{1}, d_{2}, \cdots, d_{m n}\right)$ was diagonal matrix. Eq.(19) degenerates into Eq.(14) when $v(\mathbf{x})=0$.

\subsection{Computation of Schrödinger transform}

The transfer function of Schrödinger transform of image is $e^{-i t\left(V+a|y|^{2}\right)}$. For a given $m \times n$ image and constant at, I-type Schrödinger transform of image $\varphi(x, y)$ can be computed as the following steps:

1. Suppose the low frequency component be in the center of image, we construct a $m \times n$ distance matrix $D=\left(d_{u v}\right)$, where

$$
d_{s t}=(u-m / 2)^{2}+(v-n / 2)^{2}
$$

2. Compute the transfer function of I-type Schrödinger transform, $H=\left(h_{u v}\right)$

$$
h_{u v}=e^{-a t i d_{u v}}
$$

3. Compute the Fourier transform of $\varphi(x, y), \hat{\varphi}(u, v)$ 
4. Compute the Fourier transform of $\mathbb{S}(\varphi), \hat{\mathbb{S}}(\varphi)$, according to the following formula:

$$
\hat{\mathbb{S}}(\varphi)=e^{-a i t d_{u v}} \hat{\varphi}(u, v)
$$

5. The I-type Schrödinger transform of image $\varphi(x, y)$ is the modulus of the inverse Fourier transform of $\hat{\mathbb{S}}(\varphi)$.

It is difficult to directly compute II-type Schrödinger transform of image using Eq.(19). We can compute II-type Schrödinger transform using a two step method since the matrix $V$ is Block Circulant Matrix and the matrix $|\mathbf{y}|^{2}$ is diagonal matrix in Eq.(19).

The block circulant matrix $V$ is similar to a diagonal matrix, that is,

$$
V=W D_{V} W^{-1}
$$

Here, $W=W_{m} \otimes W_{n}, W_{m}$ is a $m \times m$ matrix with $W_{m}(j, k)=e^{-2 \pi i j k / m}, W^{-1}=W_{m}^{-1} \otimes W_{n}^{-1}$, $W_{m}^{-1}(j, k)=\frac{1}{m} e^{2 \pi i j k / m}$, and $D_{V}$ is a $m n \times m n$ diagonal matrix.

We know that the exponential function satisfies $e^{x+y}=e^{x} e^{y}$ for any real numbers (scalars) $x$ and $y$. The same goes for commuting matrices: if the matrices $X$ and $Y$ commute (meaning that $X Y=Y X$ ), then $e^{X+Y}=e^{X} e^{Y}$. It is usually necessary for $A$ and $B$ to commute for the law to still hold. However, in mathematics, the Lie product formula, named for Sophus Lie, holds for all matrices $A$ and $B$, even ones which do not commute. That is, for arbitrary real or complex matrices $A$ and $B$,

$$
e^{A+B}=\lim _{N \rightarrow \infty}\left(e^{A / N} e^{B / N}\right)^{N}
$$

The formula has applications, for example, in the path integral formulation of quantum mechanics. It allows one to separate the Schrödinger evolution operator into alternating increments of kinetic and potential operators. Hence, we rewrite (19) as

$$
\hat{\mathbb{S}}_{v}(\varphi)=e^{-i t\left(V+a|\mathbf{y}|^{2}\right)} \hat{\varphi}(\mathbf{y}) \approx\left(e^{-i t V / N} e^{-i a t|\mathbf{y}|^{2} / N}\right)^{N} \hat{\varphi}(\mathbf{y})
$$

For a given $N$, denote,

$$
\hat{\varphi}_{k}(\mathbf{y})=\left(e^{-i t V / N} e^{-i a t|\mathbf{y}|^{2} / N}\right)^{k} \hat{\varphi}(\mathbf{y})
$$

So,

$$
\hat{\varphi}_{k}(\mathbf{y})=e^{-i t V / N} e^{-i a t|\mathbf{y}|^{2} / N} \hat{\varphi}_{k-1}(\mathbf{y})
$$

Where $\hat{\varphi}_{0}(\mathbf{y})=\hat{\varphi}(\mathbf{y})$.

Replacing $V$ by Eq.(21) in Eq.(25), we get that

$$
\hat{\varphi}_{k}(\mathbf{y})=W e^{-i t D_{V} / N} W^{-1} \hat{\mathbb{S}}\left(\varphi_{k-1}\right)
$$


That is,

$$
W^{-1} \hat{\varphi}_{k}(\mathbf{y})=W e^{-i t D_{V} / N} W^{-1} \hat{\mathbb{S}}\left(\varphi_{k-1}\right)
$$

Note that, $\hat{\mathbb{S}}\left(\varphi_{k-1}\right)$ are Fourier transforms of I-type of image $\varphi_{k-1}(\mathbf{x})$ and $W^{-1}$ is inverse Fourier transform. Hence,

$$
\varphi_{k}(\mathbf{x})=e^{-i t D_{V} / N_{S}}\left(\varphi_{k-1}\right)(\mathbf{x})
$$

Here, $k=1,2, \cdots, N, D_{V}$ is the inverse Fourier transform of $V$ while $V$ is the Fourier transform of $v(\mathbf{x})$, that is, $D_{V}$ is a $m n \times m n$ diagonal matrix with the diagonal elements $v(\mathbf{x})$. So, we obtain the following two step method of computing II-type Schrödinger transform:

Step 1: Using the formual $\hat{\mathbb{S}}\left(\varphi_{k-1}\right)=e^{-i t V / N} e^{-i a t|\mathbf{y}|^{2} / N} \hat{\varphi}_{k-1}(\mathbf{y})$, compute I-type Schrödinger transform of image $\varphi(x, y)$ in the frequency domain of image (see step (1) to step (4) of computing I-type Schrödinger transform of image );

Step 2: Compute II-type Schrödinger transform of image $\varphi(x, y)$ in the spatial domain of image according to Eq.(28), that is,

$$
\mathbb{S}_{v}(\varphi)(\mathbf{x}) \approx \varphi_{N}(\mathbf{x})=e^{-i t D_{V} / N_{\mathbb{S}}}\left(\varphi_{N-1}\right)(\mathbf{x})
$$

Eq.(29) means that discrete Schrödinger transform of image $\varphi(\mathbf{x})$ based on $v(\mathbf{x})$ is the approximative superposition of the following two equations

$$
\left\{\begin{array}{c}
i \cdot \overrightarrow{\hat{u}}_{t}^{I}=a|\mathbf{y}|^{2} \overrightarrow{\hat{u}}^{I} \\
\left.\overrightarrow{\hat{u}}^{I}\right|_{t=0}=\hat{\hat{\varphi}}
\end{array},\right.
$$

and

$$
\left\{\begin{array}{l}
i \cdot \overrightarrow{\hat{u}}_{t}=V \overrightarrow{\hat{u}} \\
\left.\overrightarrow{\hat{u}}\right|_{t=0}=\overrightarrow{\hat{u}}^{I^{\prime}}
\end{array},\right.
$$

Obviously, for continous Schrödinger transform of image $\varphi(\mathbf{x})$ based on $v(\mathbf{x})$, the conclusion mentioned above is not true proposition, that is, eq.(12) can not decompose the following two initial-value problem:

$$
\left\{\begin{array}{c}
i \cdot u_{t}^{I}+a \Delta u^{I}=0 \\
\left.u^{I}\right|_{t=0}=\varphi(\mathbf{x})
\end{array}\right.
$$

and

$$
\left\{\begin{array}{l}
i \cdot u_{t}=v(\mathbf{x}) u \\
\left.u\right|_{t=0}=u^{I}(\mathbf{x})
\end{array}\right.
$$

Hence, there are essential differences betwwen continous Schrödinger transform continous and discrete Schrödinger transform. 


\subsection{Properties and meaning of Schrödinger transform}

There are a variety of properties associated with the Schrödinger transform of image. The following are some of the most relevant for I-type Schrödinger transform of image. Energy conservation property also exists for the Schrödinger transform of image like the Fourier transform.

Proposition 1. (Energy Conservation Theorem)Let $u(\mathbf{x}, t)$ be Schrödinger transform of image $\varphi(\mathbf{x})$, then

$$
\int_{-\infty}^{+\infty}|u(\mathbf{x}, t)|^{2} d x=\int_{-\infty}^{+\infty}|\phi(\mathbf{x})|^{2} d x
$$

The proposition can be proved according to equation (14) and energy conservation properties of 2D Fourier Transform of image. The energy conservation properties of Schrödinger transform show that energy will diffuse from high energy to low energy while total energy is invariable(Fig. 2).

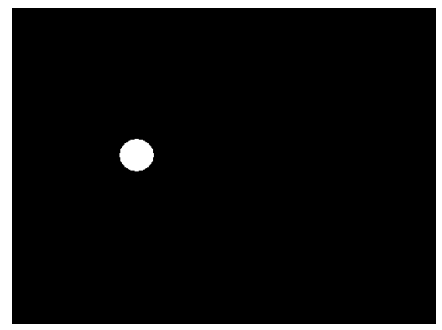

(a)

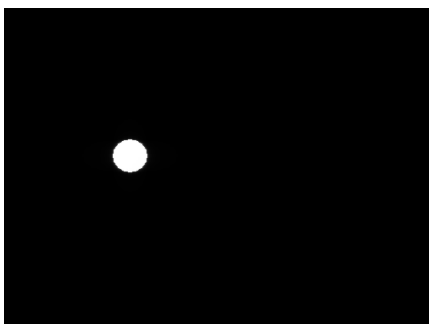

(b)

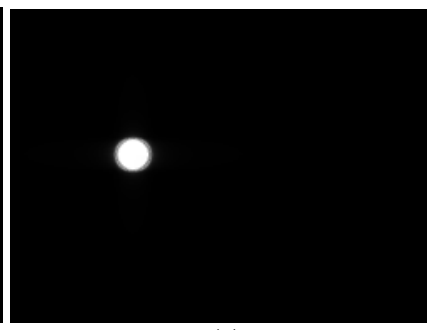

(c)

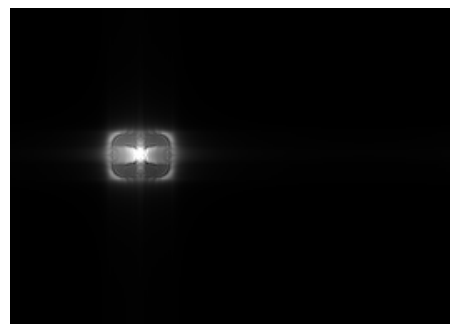

(d)

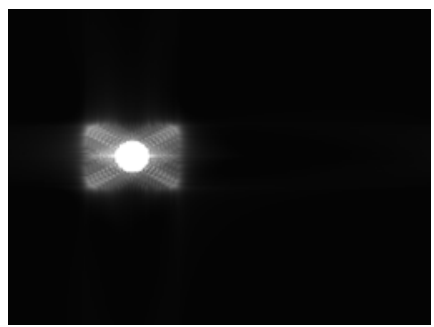

(e)

(a)original image, (b), (c), (d) and (e) are Schrödinger transforms with parameters 0.00001, 0.00005, 0.0005 and 0.001 , respectively.

Fig. 2. Schrödinger transforms of a circular disc image with different parameters at

Proposition 2. Let filter transfer function be $H(\mathbf{y})=\frac{1}{2}\left(1+e^{-a i t|\mathbf{y}|^{2}}\right)$, where $\mathbf{y}=\left(\begin{array}{l}y_{1} \\ y_{2}\end{array}\right)$, $|\mathbf{y}|^{2}=y_{1}^{2}+y_{2}^{2}$, If $|\mathbf{y}|^{2} \leq M$ (There exists highest frequency for digital image), then $|H(\mathbf{y})|^{2}$ is a decrease function of $|\mathbf{y}|^{2}$ when $a t \leq \frac{\pi}{2 M}$, that is, $H(\mathbf{y})$ is low pass filter. 
Proposition 3. Let filter transfer function be $H(\mathbf{y})=1-e^{-a i t|\mathbf{y}|^{2}}$, where $\mathbf{y}=\left(\begin{array}{l}y_{1} \\ y_{2}\end{array}\right)$, $|\mathbf{y}|^{2}=y_{1}^{2}+y_{2}^{2}$, If $|\mathbf{y}|^{2} \leq M$ (There exists highest frequency for digital image), then $|H(\mathbf{y})|^{2}$ is a increase function of $|\mathbf{y}|^{2}$ when $a t \leq \frac{\pi}{2 M}$, that is, $H(\mathbf{y})$ is high pass filter.

High and low pass filter can be obtained using Schrödinger transform of image according to the above two propositions. And Schrödinger transform of image can be applied to image processing and analysis, such as, boundary extraction, edge enhancement, etc.

The energy conservation properties of Schrödinger transform show that energy will diffuse from high energy to low energy while total energy is invariable(Fig. 2).The following experiments (see Fig. 3 and Fig. 4) show the meaning and function of Schrödinger transformation of image, that is, Schrödinger transformation of image can be seen as the result of primitive image shrinking inside and spreading outward at the center of object, like as interference wave. The bigger at is, the more obvious the interference is. On the other hand, contour curves of object in the transformed image are similar to contour curves of object in the original image, and they are too similar to draw them manually. Hence, I-type Schrödinger transform of image is isotropic.
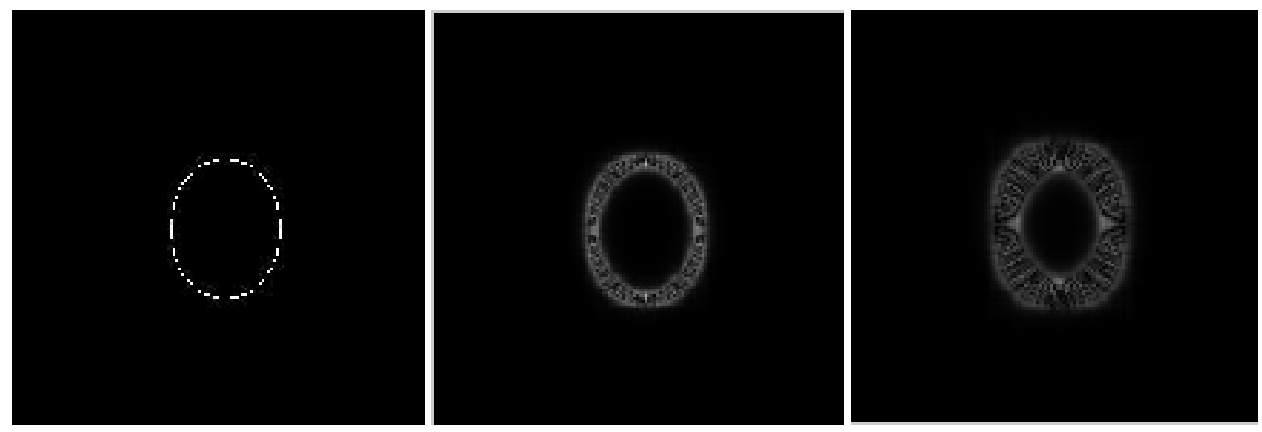

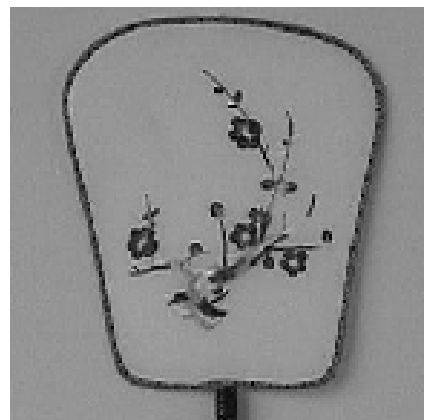

(a)

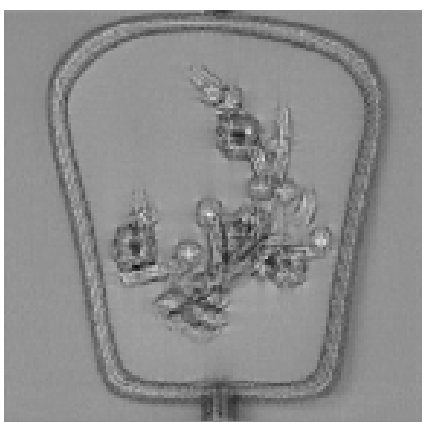

(b)

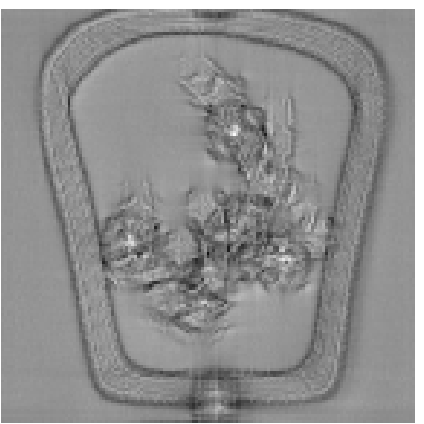

(c)

Fig. 3. I-type Schrödinger transform of image. (a) The original image, (b), (c) are Schrödinger transforms. The constant at is $0.0005,0.001$, respectively. 


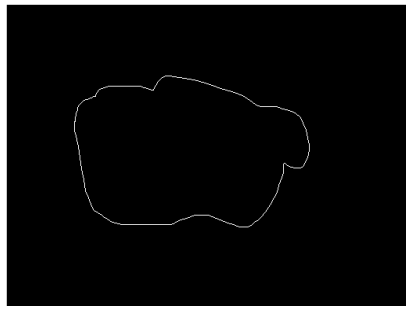

(a)

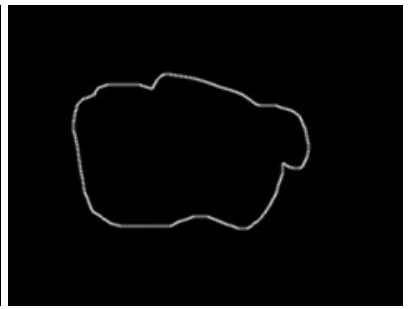

(b)

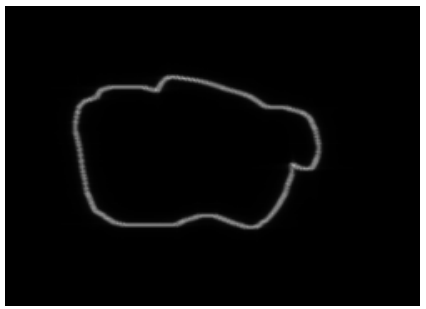

(c)

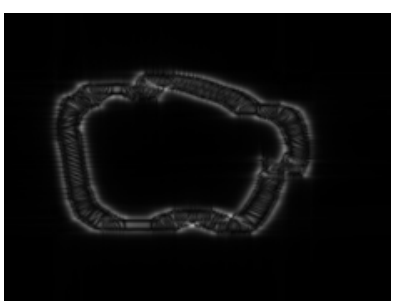

(d)

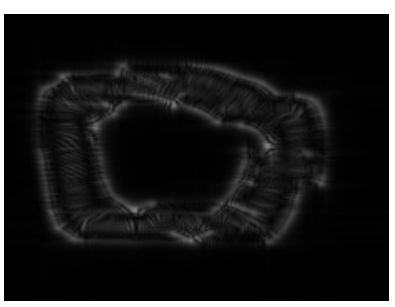

(e)

(a)original image, (b), (c), (d) and (e) are Schrödinger transforms with parameters 0.00001, 0.00005, 0.0005 and 0.001 , respectively.

Fig. 4. Schrödinger transforms of a irregular closed image with different parameters at

\section{Scale parameter and potential function of Schrödinger transform}

It is important to select the parameter at of Schrödinger transformation while the potential function is selecte. In the section, we'll discuss the effects of scale parameter and potential function for Schrödinger Transform of image.

\subsection{Schrödinger transform of rectangle image}

Fig. 2 shows the parameter at is scale parameter of Schrödinger transformation. To make clear the relation between the parameter at and Schrödinger transformation, without loss of generality, take Schrödinger transform of rectangle image for example. And, the parameter at is denoted by the parameter $a$.

Let a rectangle image be

$$
f(m, n)=\left\{\begin{array}{l}
1, \text { if } M_{1} \leq m \leq M_{2}, N_{1} \leq n \leq N_{2} \\
0, \text { otherwise }
\end{array}\right.
$$

Its Fourier transform is

$$
\begin{gathered}
F(u, v)=\sum_{m=M_{1}}^{M_{2}} \sum_{n=N_{1}}^{N_{2}} \exp \left(-j 2 \pi\left(\frac{m u}{M}+\frac{n v}{N}\right)\right) \\
=\frac{\left(e^{-j 2 \pi u M_{1} / M}-e^{-j 2 \pi u\left(M_{2}+1\right) / M}\right)\left(e^{-j 2 \pi v N_{1} / N}-e^{-j 2 \pi v\left(N_{2}+1\right) / N}\right)}{\left(1-e^{-j 2 \pi u / M}\right)\left(1-e^{-j 2 \pi v / N}\right)}
\end{gathered}
$$


The transfer function of two-dimensional discrete Schrödinger transform is

$$
H(u, v)=e^{-j a\left((u-M / 2)^{2}+(v-N / 2)^{2}\right)}
$$

$$
\begin{aligned}
& G(u, v)=F(u, v) H(u, v) \\
& =\frac{\left(e^{-j 2 \pi u M_{1} / M}-e^{-j 2 \pi u\left(M_{2}+1\right) / M}\right) e^{-j a(u-M / 2)^{2}}}{1-e^{-j 2 \pi u / M}} \times \frac{\left(e^{-j 2 \pi v N_{1} / N}-e^{-j 2 \pi v\left(N_{2}+1\right) / N}\right) e^{-j a(v-N / 2)^{2}}}{1-e^{-j 2 \pi v / N}}
\end{aligned}
$$

Then, Schrödinger transform of rectangle image $f(m, n)$ is

$$
\begin{gathered}
g(m, n)=\frac{1}{M N} \sum_{u=0}^{M-1} \sum_{v=0}^{N-1} G(u, v) \exp \left(j 2 \pi\left(\frac{m u}{M}+\frac{n v}{N}\right)\right) \\
=\frac{1}{M} \sum_{u=0}^{M-1} \frac{\left(e^{-j 2 \pi u M_{1} / M}-e^{-j 2 \pi u\left(M_{2}+1\right) / M}\right) e^{-j a(u-M / 2)^{2}} e^{j 2 \pi m u / M}}{1-e^{-j 2 \pi u / M}} \\
\times \frac{1}{N} \sum_{v=0}^{N-1} \frac{\left(e^{-j 2 \pi v N_{1} / N}-e^{-j 2 \pi v\left(N_{2}+1\right) / N}\right) e^{-j a(v-N / 2)^{2}} e^{j 2 \pi n v / N}}{1-e^{-j 2 \pi v / N}} \\
=g_{M, M_{1}, M_{2}}(m) g_{N, N_{1}, N_{2}}(n) \\
\text { Here } g_{M, M_{1}, M_{2}}(m)=\frac{1}{M} \sum_{k=M_{1}}^{M_{2}} \sum_{u=1}^{M} e^{-j 2 \pi u(k-m) / M} e^{-j a(u-M / 2)^{2}} .
\end{gathered}
$$
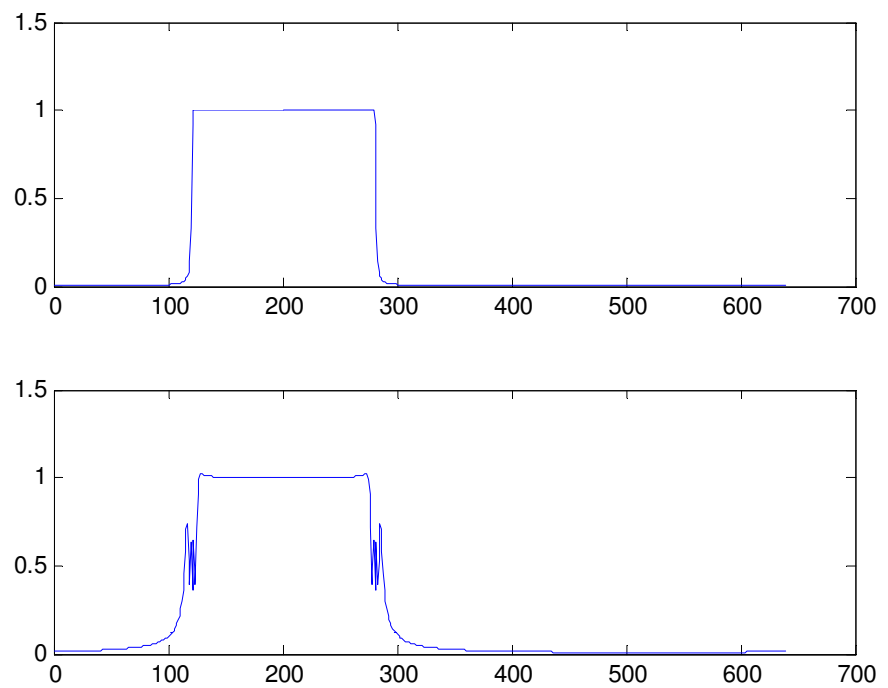

Fig. 5. The function $\left|g_{640,120,280}(m)\right| . a=0.00001$ and 0.0001 , respectively 


\subsection{Scale parameter of Schrödinger transform}

For given threshold $T(=0.5)$, denote

$$
d=\frac{1}{2}\left(M_{1}+1-\min \left\{m: g_{M, M_{1}, M_{2}}(m)>T\right\}+\max \left\{m: g_{M, M_{1}, M_{2}}(m)>T\right\}-M_{2}\right)
$$

Then, the vaiable $d$, which depend on the constant $a$, represents the propagation distance of I-type Schrödinger transform(see Fig. 6). The following experiment shows that the bigger

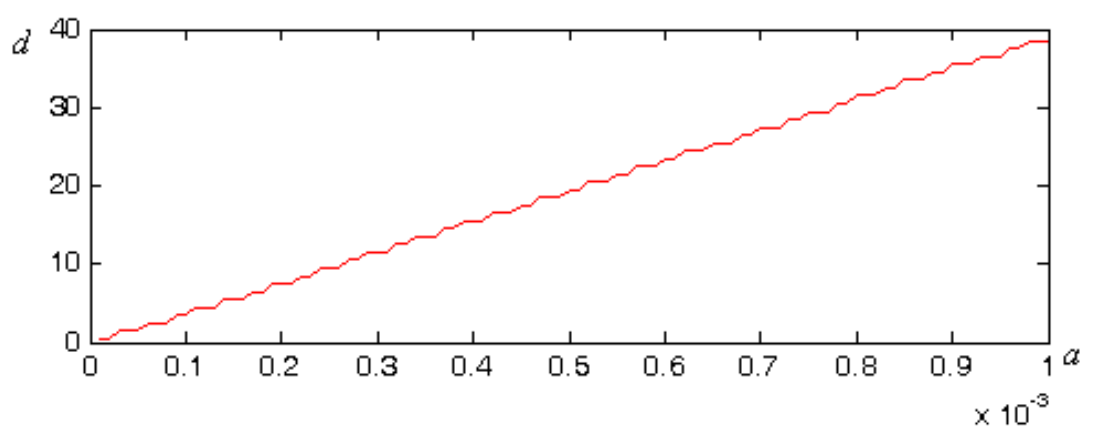

(a) $M=480, M_{1}=120, M_{2}=280, T=0.5$

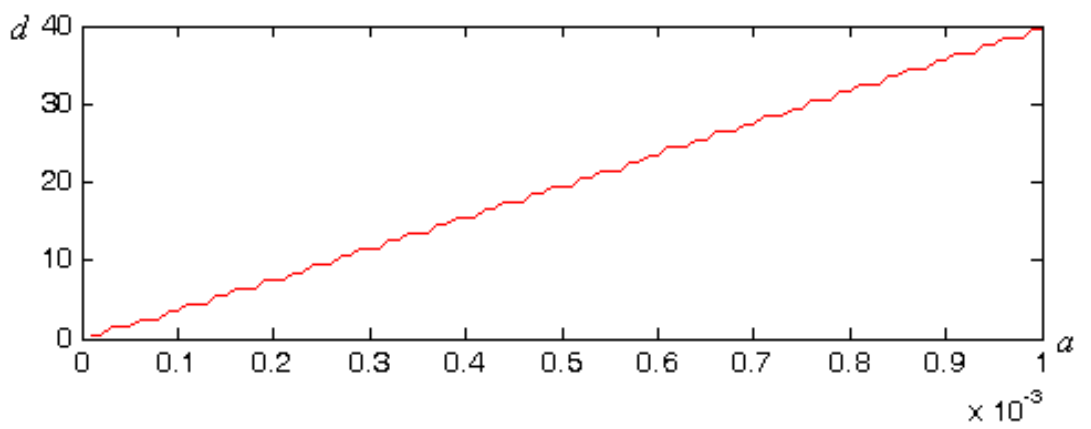

(b) $M=480, M_{1}=180, M_{2}=400, T=0.5$

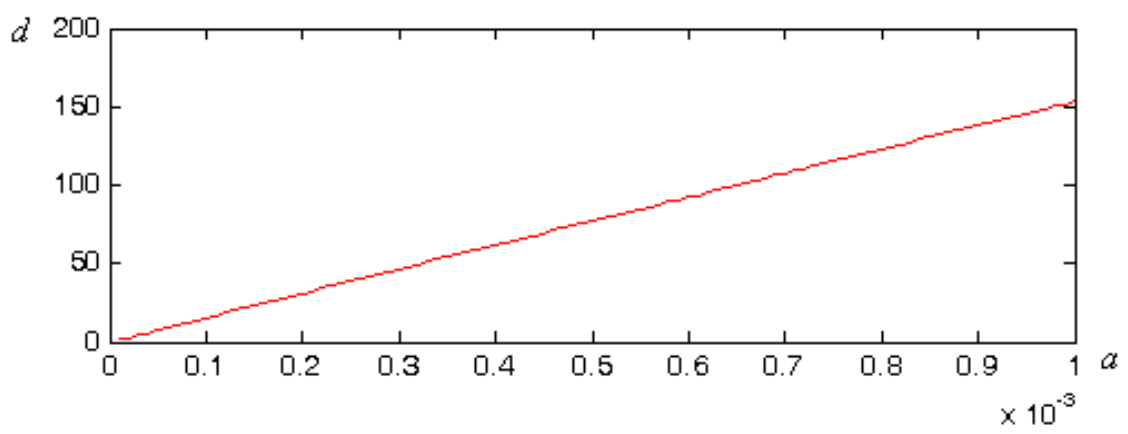

(c) $M=960, M_{1}=180, M_{2}=400, T=0.5$

Fig. 6. The relationship between propagation distance $d$ and scale parameter $a$. 
$a$ is, statistically speaking, the bigger $d$ is. So, the constant $a$ (or at) is the Scale Parameter of Schrödinger Transform. In the experiment, we investigate the relation between propagation distance and the constant $a$, size of image $M$, size of object $M_{2}-M_{1}$. The variable $M$ varies from 480 to 900 with the increment 10, $M_{2}$ from $M_{1}+10$ to $M-100$ with the increment 2, and $a$ from 0.00001 to 0.0001 with the increment 0.00001 while $M_{1}=170$ and the distance threshold $T=0.5$. We can obtain 88580 samples using Equation (37). The Table 1 lists some descriptive statistics of sample datas. Pearson correlation coefficents between propagation distance $d$ and $a, M, M_{2}-M_{1}$ is $0.823,0.494$ and 0.224 , respectively. The multiple regression procedures of SPSS estimate a linear equation of the form:

$$
d=-9.625+0.13 M+0.01\left(M_{2}-M_{1}\right)+90249.047 a
$$

And standard error of the estimate, which is less than one pixel, is 0.88556 . From Fig. 6 and Equation (38), we know the biggest affect on propagation distance $d$ is the scale parameter $a$, size of image $M$ takes second place while the propagation distance $d$ and scale parameter $a$ have a strong positive linear correlation, the propagation distance $d$ and size of image $M$ have a weak positive linear correlation,.

\begin{tabular}{|l|l|l|}
\hline variable & Mean & Std. Deviation \\
\hline$d$ & 4.8186 & 3.15129 \\
\hline$M$ & 727.38 & 118.334 \\
\hline$M_{2}-M_{1}$ & 233.69 & 146.615 \\
\hline$a$ & .00005500 & .000028723 \\
\hline
\end{tabular}

Table 1. Descriptive Statistics

Remark: Schrödinger Transform of image can be directly computed for a little number $a$ by using Equation (14) while it needs to use Equation (14) repeatedly for a big unmber $a$, and use a little scale parameter every times, so that interference effect of Schrödinger Transform can be avoided for using a big scale parameter $a$.

\subsection{Potential function of Schrödinger transform}

It doesn't need to use potential function for I-type Schrödinger Transform of image, which is isotropic. II-type Schrödinger Transform is anisotropic since nonzero potential function is applied. It is necessary that a right potential function is chosed so that we can obtain a perfect deformation processing according to anisotropic property of II-type Schrödinger Transform. However, if the potential function $D_{V}$ is real, from Equation (29), we know

$$
\left|\mathbb{S}_{V}(\varphi)(\mathbf{x})\right| \approx\left|\varphi_{N}(\mathbf{x})\right|=\left|e^{-i t D_{V} / N_{S}} \mathbb{S}\left(\varphi_{N-1}\right)(\mathbf{x})\right|=\left|\mathbb{S}\left(\varphi_{N-1}\right)(\mathbf{x})\right|
$$

That is, II-type Schrödinger Transform of image $\varphi(\mathbf{x})$ based on $v(\mathbf{x}), \mathbb{S}_{v}(\varphi)$, is only equivalent to implement I-type Schrödinger Transform $N$ times for image $\varphi$. Obviously, that is surely not the result we want. So, the potential function $D_{V}$ of II-type Schrödinger Transform must be imaginary so that $\left|e^{-i t D_{V} / N}\right| \neq 1$. Meanwhile, if we hope that deformation 
processing of II-type Schrödinger Transform stop in some place, $\left|e^{-i t D_{V} / N}\right|$ will be small in the place. Hence, if we hope that deformation processing of II-type Schrödinger Transform will be carried through in homogeneous region of image while it will stop in edge region of image, we can select the potential function of I-type Schrödinger Transform as the following formula:

$$
D_{V}=-i h(G(v(\mathbf{x})))
$$

Where $G(\cdot)$ is arbitrary gradient or edge-detection operator, $h()$ is a monotone increasing function.

\section{Application of Schrödinger transform}

As a new tool for image analysis, Schrödinger Transform of image can be applied to image smoothing, image enchancement, contour extraction, image inpainting and so on. And Schrödinger Transform of image can also be applied to image segmentation for 3D image. In the section, we'll give some examples.

5.1 Constructing high and low pass filter using l-Type Schrödinger transform of image By the definition and formula of Schrödinger transform of image, transformed image has the same size as the original image. In transformed image, every pixel is related to that of all pixels of original image. So Schrödinger transform is global. High and low pass filter of image, which can detect image edge, enhance and smooth image, can be constructed according to Property 2 and 3 of Schrödinger transform. The filter consider both global and local feature of image while the traditional edge detection operators use only local template.

Suppose Schrödinger transform of $m \times m$ image $\varphi(\mathbf{x})$ be $u(\mathbf{x}, t)$. Then, $\frac{1}{2}|\phi(\mathbf{x})+u(\mathbf{x}, t)|$ is the smoothed image of image $\varphi(\mathbf{x})$ while $|\phi(\mathbf{x})-u(\mathbf{x}, t)|$ is the edge image of $\varphi(\mathbf{x})$ if $a t \leq \frac{\pi}{2 m^{2}}$. However, $u(\mathbf{x}, t)$ is a complex-valued image, the result is not good using the above high and low pass filtering directly. Replacing $|\phi(\mathbf{x})-u(\mathbf{x}, t)|$ with $|\phi(\mathbf{x})-| u(\mathbf{x}, t)||$ has better high pass filtering effect. Similarly, the smoothing effect of $\frac{1}{2}|\phi(\mathbf{x})+| u(\mathbf{x}, t)||$ is better than $\frac{1}{2}|\phi(\mathbf{x})+u(\mathbf{x}, t)|$. The steps of high pass filtering of image by Schrödinger transform are as follows(the steps of low-pass filtering is similar):

1. Compute Fourier transform $\phi(\mathbf{y})$ of image $\varphi(\mathbf{x})$;

2. Compute Fourier transform $u(\mathbf{y}, t)$ of $u(\mathbf{x}, t)$, according to $u(\mathbf{y}, t)=\phi(\mathbf{y}) e^{-a i t|\mathbf{y}|^{2}}$;

3. Compute inverse Fourier transform $u(\mathbf{y}, t)$ to obtain Schrödinger transform $u(\mathbf{x}, t)$ of image $\varphi(\mathbf{x})$;

4. Compute $|\phi(\mathbf{x})-| u(\mathbf{x}, t)||$ to obtain high pass filtered image;

5. Enhancing edge of image $\varphi(\mathbf{x})$ by $\phi(\mathbf{x})+|\phi(\mathbf{x})-| u(\mathbf{x}, t) \mid$. 
We should pay attention to two issues using the above method to detect image edge, enhance image and smooth image:

1. The parameter at should be appropriate. If the parameter is too small ,the filtering effect would not be obvious. Contrariwise, Schrödinger transforme will cause interference which effects the filtering. The parameter at should not exceed $\frac{\pi}{2 M^{2}}$. In the following experiments, Schrödinger transform of image is completed by using Schrödinger transform with smaller at repeatly So that the interference can be avoided.

2. The origin of coordinates of frequency domain is the center rather than the top left corner of the image when the formual $u(\mathbf{y}, t)=\phi(\mathbf{y}) e^{-a i t|\mathbf{y}|^{2}}$ is used to compute Fourier transform $u(\mathbf{y}, t)$ of $u(\mathbf{x}, t)$.

We give the results of image edge detection by using Schrödinger Transform in Fig. 7. The experements show the comparison results of a fan image by several edge detection operators in Fig. 8. According to the comparison results of traditional edge detection operators, the high-pass filter designed by Schrödinger transform can better detect image edge and it would not increase noise simultaneously. In fact, filter designed by Schrödinger transform consider both the local and global feature of image, so filtering effect is better.
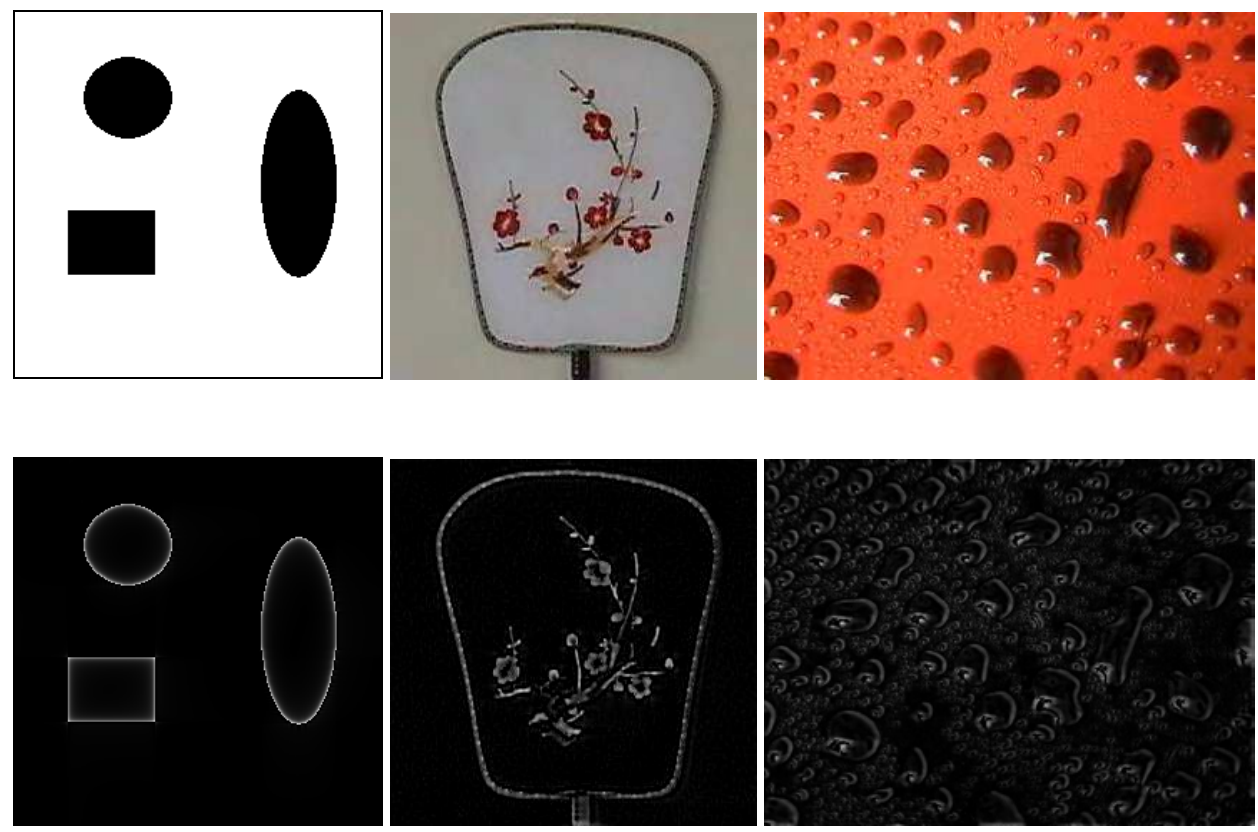

Fig. 7. Edges detected using Schrödinger Transforms of image 


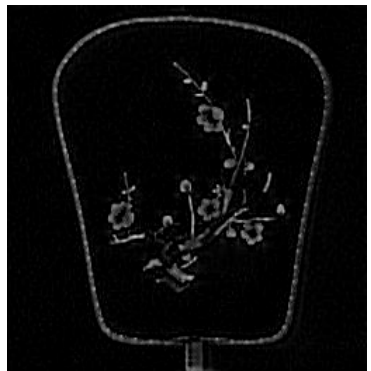

(a)

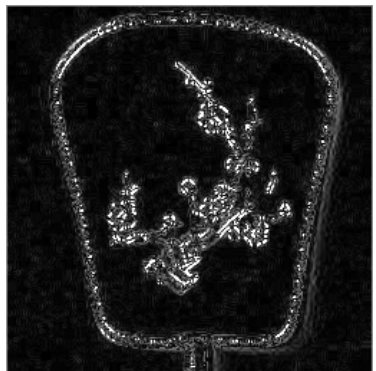

(d)

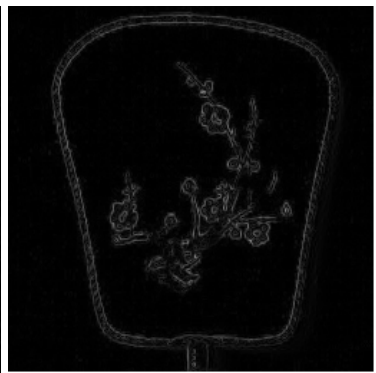

(b)

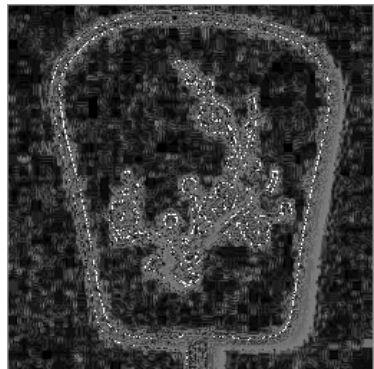

(e)

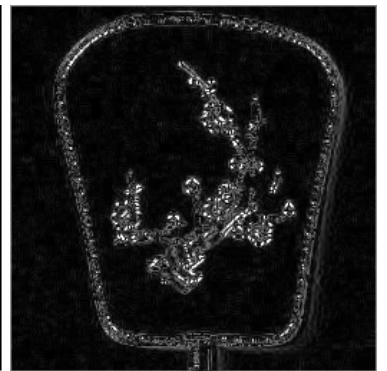

(c)

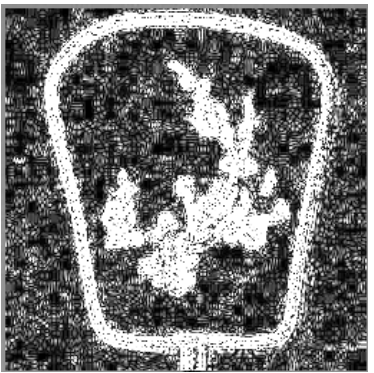

(f)

(a) High pass filter based on Schrödinger Transform of Image, (b) Robert operator, (c) Prewitt operator, (d) Sobel operator, (e) Canny operator, (f) Laplace operator

Fig. 8. Comparison of several edge detection methods

\subsection{Automatic contour extraction for multiple objects using I-Type Schrödinger transform of image \\ 5.2.1 Exterior and interior points of multiple objects}

Assume $I(x, y)$ be an original image that contains multiple dark objects. We use Schrödinger Transforms of original image $I(x, y)$ and its inverse image $255-I(x, y)$ to obtain exterior and interior of multiple objects. So we can separate dark objects from bright background. Let $u_{1}(\mathbf{x}, t), u_{2}(\mathbf{x}, t)$ be Schrödinger transforms of original image and its inverse image, respectively. To obtain enough exterior and interior points exactly, in the following experiments, we implement Schrödinger transforms of image many times using small parameter at. Denote

$$
\begin{gathered}
I_{1}(x, y)=\left|I(x, y)-u_{1}(\mathbf{x}, t)\right| \\
I_{2}(x, y)=\left|255-I(x, y)-u_{2}(\mathbf{x}, t)\right|
\end{gathered}
$$

Then, according to the Proposition 1, these pixels with high gray values in the image $I_{1}(x, y)$ are interior points of multiple objects while these pixels with high gray values in the image $I_{2}(x, y)$ are exterior points of multiple objects. To obtain interior and exterior points of multiple objects, we convert an intensity image to a binary image using Otsu's threshold method. 


\subsubsection{Contour extraction of multiple objects}

After obtaining exterior and interior points of multiple objects, we take the following steps to extract contours of multiple objects:

1. Thinning of sets of exterior and interior points.

Sets of exterior and interior points need to be thinned because there are too many points in the two sets. Generally speaking, the exterior points surround the interior points for the same object. For each point $A$ in the set of exterior points(or interior points), we could find the point $B$ which is the point with the shortest distance between the point $A$ and the set of interior points(or exterior points). The point $B$ shall be added into the thinned set of interior points(or exterior points).

2. Find pairs of exterior and interior points with the smallest distance between them.

The closest pair of points problem is a problem of computational geometry. Using the brute-force algorithm, the closest pair of points can easily be computed in $O\left(n^{2}\right)$ time. To do that, one could compute the distances between all pairs of points, then pick the pair with the smallest distance. The problem can also be solved in $O(n \log n)$ time using the recursive divide and conquer approach (Cormen et al., 2001).

\subsubsection{Algorithm}

The actual operation of the algorithm is as follow:

1. If necessary, convert an original gray image to a binary image using Otsu's threshold method.

2. Compute Schrödinger transforms $u_{1}(\mathbf{x}, t), u_{2}(\mathbf{x}, t)$ of original image and its inverse image.

3. Compute $I_{1}(x, y)=\left|I(x, y)-u_{1}(\mathbf{x}, t)\right|, I_{2}(x, y)=\left|255-I(x, y)-u_{2}(\mathbf{x}, t)\right|$.

4. Find interior and exterior points of multiple objects by converting images $I_{1}(x, y)$ and $I_{2}(x, y)$ to binary images using Otsu's threshold method.

5. Thin sets of exterior and interior points.

6. Find pairs of exterior and interior points by using the brute-force algorithm. If the distance between the pair points is larger than some constant, a new object will appear.

7. Obtain contours of multiple objects by connecting all pairs of points belong to the same object in turn.

\subsubsection{Experiments}

To evaluate the performance of proposed scheme by experiments, we do Schrödinger transform and contour extraction experiments using the simulated and real images. Schrödinger transform of image can separate boundaries of object from background. Contour extraction experiments for the simulated images consisting of three objects are shown in Fig. 9. With growing pollution levels and an ever growing coastal population, hydrophobicity (water repellence) of insulators has become an important consideration for equipment specifiers. An insulator with hydrophobic properties causes water to bead on its surface and roll off assisting pollution to freely wash away rather than forming a continuous wet sheet or zone and combining with pollutants to form a conductive film. The hydrophobic effect helps to reduce discharge activity and maintenance requirements as well as decreasing the probability of flashover. Hydrophobicity demonstrated by beading on the surface of a new polymer insulator(see Fig. 10(a)). The extracted contours of beads using Schrödinger transform of image are shown in Fig. 10(d). 


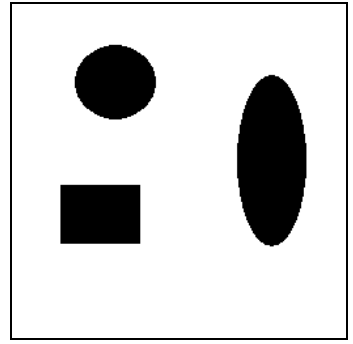

(a)

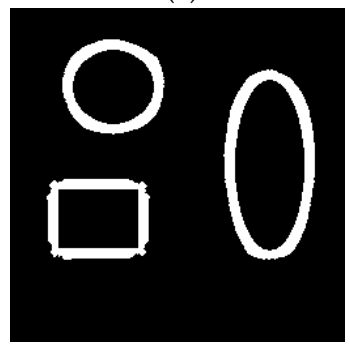

(c)

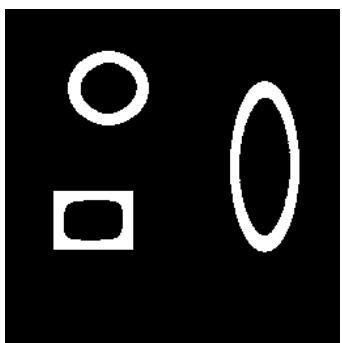

(b)

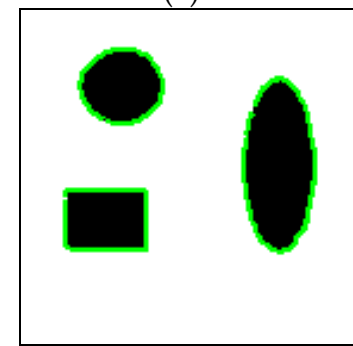

(d)

(a) The original simulated image, (b) The interior points of objects (white pixels),

(c) The exterior points of objects (white pixels), (d) the extracted contours.

Fig. 9. The extracted contours of multiple objects using Schrödinger transform of image.

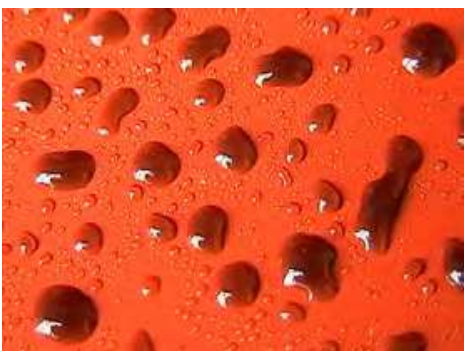

(a)

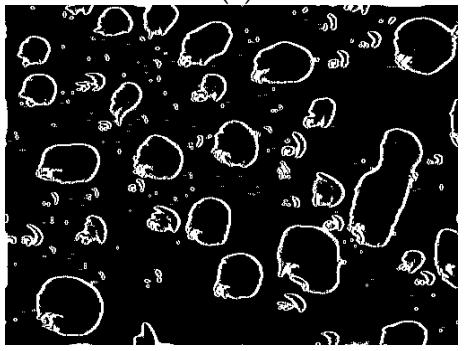

(c)

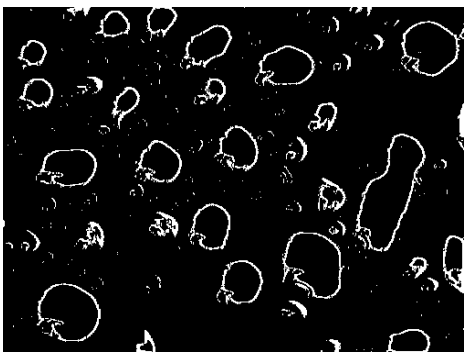

(b)

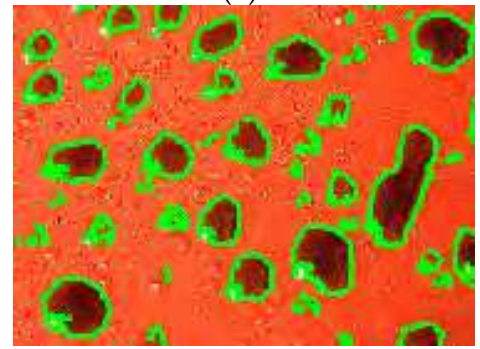

(d)

(a) beads on the surface of a polymer insulator, (b) The interior points of objects (white pixels),

(c) The exterior points of objects (white pixels), (d) The extracted contours of beads.

Fig. 10. The extracted contours of beads using Schrödinger transform of image. 


\subsection{Image segmentation using II-Type Schrödinger transform of image}

The following experiments show how to segment the target area by useing II-Type Schrödinger Transform. Fig. 11 shows segmentation results of an image with three goals, Fig. 12 shows object segmentation results of a fan image. The two results show that we can extract the contour of object using evolution of II-Type Schrödinger Transform of image. The segmentation results are less depending on the initial contour, as long as be the inside or outside the target area.

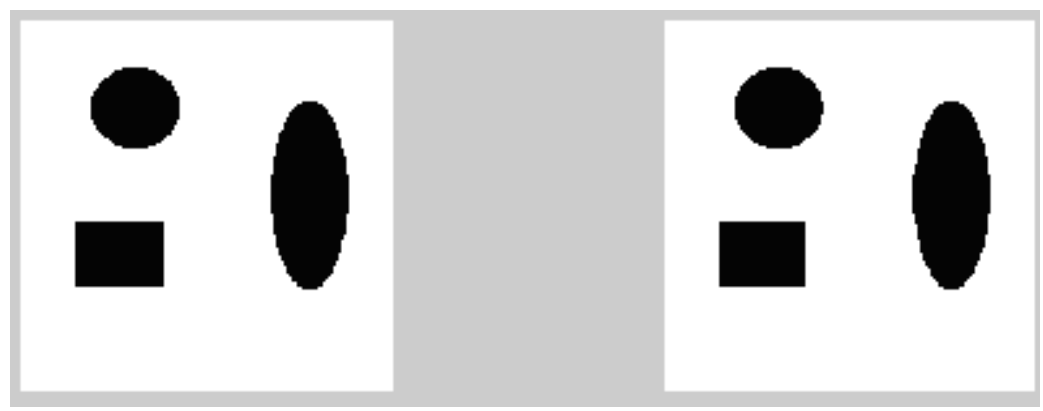

(a)

(b)

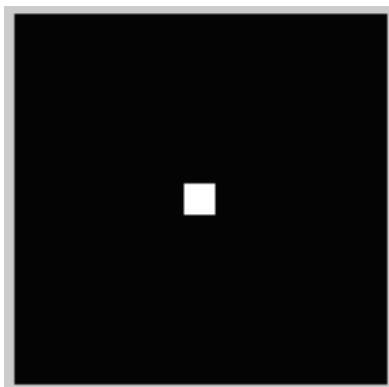

(c)

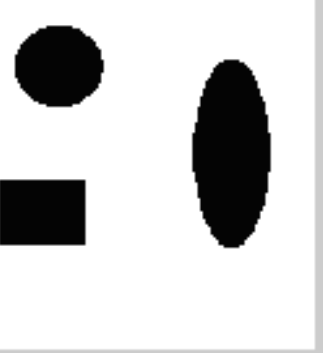

(d)

(a) original image, (b) image of potential function,

(c) initial region of object, (d) segmented region of object

Fig. 11. segmented result images of three objects by using II-type Schrödinger transform of image 

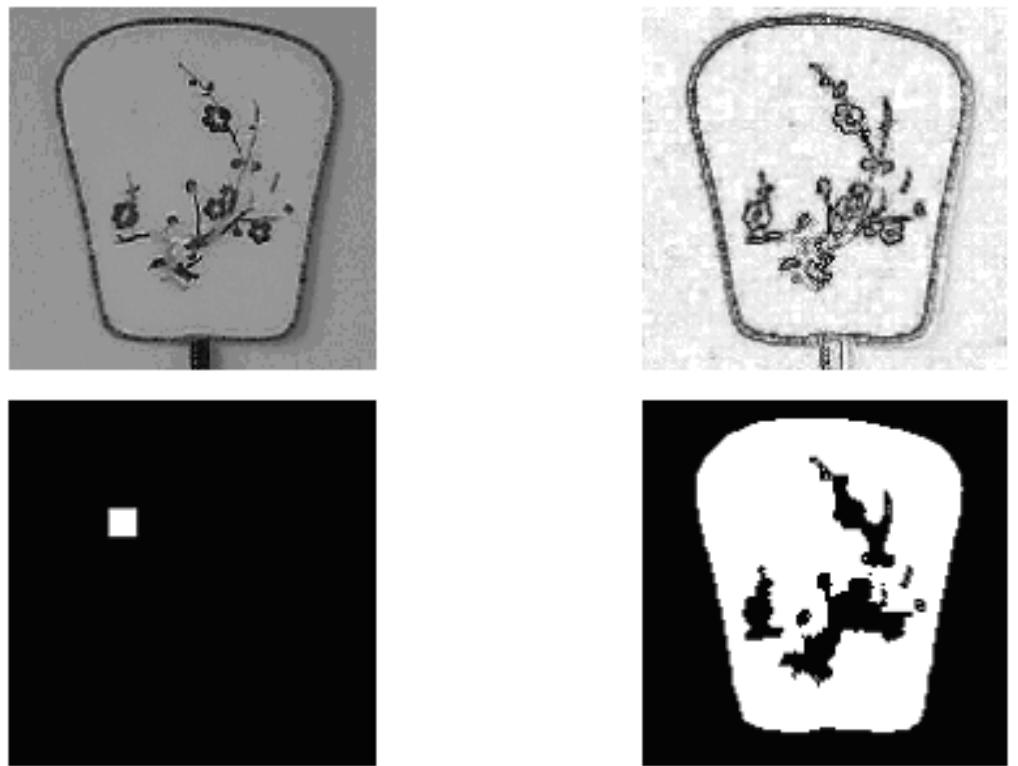

(a) original image, (b) image of potential function,

(c) initial region of object, (d) segmented region of object

Fig. 12. segmented result of fan image by using II-type Schrödinger transform of image

\section{Conclusion}

Image segmentation has been, and currently still is, a relevant research area in Computer Vision, and hundreds of segmentation algorithms have been proposed in the last few decades. However, it is well known that elemental segmentation techniques based on edge or region information often fail to produce accurate segmentation results. The chapter attempts to provide a brief introduction of Schrödinger transform of image for the novel segmentation techniques and discussed its potential applications in image analysis and understanding. Schrödinger transform of image is extended from I-type to II-type. Schrödinger Transform of image is applied to image smoothing, image enchancement, contour extraction, and so on. The demonstrate experiment results show the robust of the algorithm.

\section{Acknowledgment}

This work was supported by the National Natural Science Foundation of China under Grant No. 60975011. The research work was also partially supported by the Basic and Frontier Technology Research Programs of the Department of Science \& Technology of Henan Province (No. 092300410043), and Supported by Foundation for University Youths Key Teacher by the Education Department of Henan Province; and a grant from the Nature Science Basic Research Programs of the Education Department of Henan Province (No. 2007510023), and Supported by Foundation for key project by the Education Department of Hubei Province(D20101502), China. 


\section{References}

Caselles, V.; Catte, F.; Coll, T. \& Dibos, F. (1993). A Geometric model for active contours. Numer. Math., Vol.66, (1993), pp. 1-31, ISSN 0945-3245

Caselles,V.; Kimmel, R. \& Sapiro, G. (1995). Geodetic active contours. Proc. 5th Intl. Conf. Computer Vision, pp.694-699, ISBN 0-8186-7042-8, Massachusetts Institute of Technology, Cambridge, Massachusetts, USA, June 20-23, 1995

Chan, T \& Vese, LA. (1999). An active contour model without edges, Proceedings of the Second International Conference on Scale-Space Theories in Computer Vision, pp. 141151, ISBN 354066498X, Corfu, Greece, September 26-27, 1999

Chan, T \& Vese, LA. (2002). Active contour and segmentation models using geometric PDE's for medical imaging, In: Geometric methods in bio-medical image processing, Ravikanth Malladi (Editor), pp. 63-75, Springer, ISBN 9783540432166. Berlin, German

Cohen, L. D. \& Cohen, I. (1993). Finite-element methods for active contour models and balloons for 2-D and 3-D images. IEEE Trans.Pattern Anal. Mach. Intell., Vol.15, No.11, (1993), pp. 1131-1147, ISSN 0162-8828

Cohen, L. D. \& Kimmel, R. (1997). Global minimum for active contour models: a minimal path approach. Int. J. Comput. Vis.,Vol. 24, No.1, (1997), pp. 57-78, ISSN 0920-5691

Cohen, L. D. (1991). On active contour models and balloons. CVGIP: ImageUnderstand., Vol.53, No.2, (1991), pp. 211-218, ISSN 1049-9660

Cormen, T. H.; Leiserson, C. E.; Rivest, R. L. \& Stein, C. (2001). Introduction to Algorithms (Second Edition), MIT Press and McGraw-Hill, ISBN 0-262-03293-7., Cambridge, England

Evans, L. C. (1998). Partial Differential Equations, American Mathematical Society, ISBN 0821807722, Providence, USA

Feynman, R. P. \& Hibbs,A. R. (1965). Quantum Mechanics and Path Integrals, McGraw-Hill Inc., ISBN 0070206503, New York, USA

Kass, M.; Witkin,A. \& Terzopoulos,D. (1987). Snakes: active contour models. Int. J. Comput. Vis., Vol.1, No.4, (1987), pp. 321-331, ISSN 0920-5691

Lobergt, S. \& Viegever, M. A. (1995). A discrete dynamic contour model. IEEE Trans. Med. Imaging, Vol.14, No.1, (1995), pp. 12-24, ISSN 0278-0062

Lou, Liantang \& Ding, Mingyue (2007a). Principle and approach of boundary extraction based on particle motion in quantum mechanics. Optical Engineering, Vol.46, No,2, (2007), pp. 027005-1- 027005-16, ISSN 0091-3286

Lou, Liantang; Fu, ZhongLiang \& Jiang, Si (2007b). Maximal probability method of boundary extraction based on particle motion. Proc. SPIE, MIPPR 2007, pp. 67861F.1-67861F.5, ISBN 9780819469502, Wuhan, Hubei, China, November 15-17, 2007

Lou, Liantang; Zhan, Xin; Fu, Zhongliang \& Ding, Mingyue (2008). Method of Boundary Extraction Based on Schrödinger Equation, Proceedings of the 21th Congress of the International Society for Photogrammetry and Remote Sensing - ISPRS 2008, B5-2, pp. 813-816, ISSN 1682-1750, Beijing, China, July 3-11, 2008

Malladi, R. \& Sethian, JA. (1996). Level set and fast marching methods in image processing and computer vision, Proceedings of the IEEE International Conference on Image Processing, Vol.1, pp. 489 - 492, ISBN 0-7803-3259-8, Lausanne, Switzerland, Sep., 16-19, 1996 
McInerney, T. \& Terzopoulos, D. (1999). Topology adaptive deformable surfaces for medical image volume segmentation. IEEE Trans. Med. Imaging, Vol.18, No.10, pp. 840-850, (1999), ISSN 0278-0062

Osher, S. \& Sethian, J. (1988). Fronts propagating with curvature dependent speed: algorithms based on Hamilton-Jacobi formulations. J. Comput Phys., Vol.79, No.1, pp. 12-49, (1988), ISSN

Terzopoulos, D.; Witkin, A. \& Kass, M. (1987). Constraints on deformable models: recovering 3D shape and nonrigid motion. Artif. Intell., Vol,36, No.1, (1987), pp. 91123, ISSN 0004-3702

Williams, L.R. \& Jacobs, D.W. (1995). Stochastic Completion Fields: A Neural Model of Illusory Contour Shape and Salience, Proc. 5th Intl. Conf. Computer Vision, pp.694699, ISBN 0-8186-7042-8, Massachusetts Institute of Technology, Cambridge, Massachusetts, USA, June 20-23, 1995

$\mathrm{Xu}, \mathrm{CY}$ \& Prince, JL. (1998). Snakes, shapes, and gradient vector flow. IEEE Trans Image Process, Vol.7, No.3, pp. 359-369, (1998), ISSN 


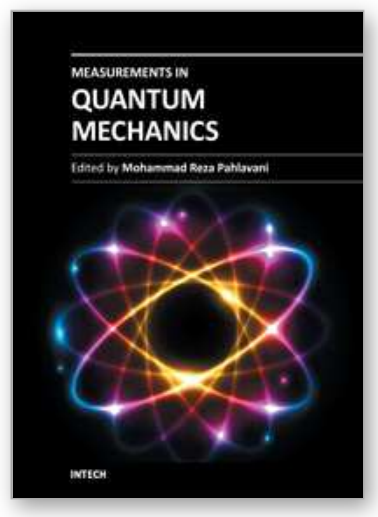

\author{
Measurements in Quantum Mechanics \\ Edited by Prof. Mohammad Reza Pahlavani
}

ISBN 978-953-51-0058-4

Hard cover, 348 pages

Publisher InTech

Published online 22, February, 2012

Published in print edition February, 2012

Perhaps quantum mechanics is viewed as the most remarkable development in 20th century physics. Each successful theory is exclusively concerned about "results of measurement". Quantum mechanics point of view is completely different from classical physics in measurement, because in microscopic world of quantum mechanics, a direct measurement as classical form is impossible. Therefore, over the years of developments of quantum mechanics, always challenging part of quantum mechanics lies in measurements. This book has been written by an international invited group of authors and it is created to clarify different interpretation about measurement in quantum mechanics.

\title{
How to reference
}

In order to correctly reference this scholarly work, feel free to copy and paste the following:

Liantang Lou, Hua Zeng, Jipeng Xiong, Lingling Li and Wenliang Gao (2012). Schrödinger Transform of Image: A New Tool for Image Analysis, Measurements in Quantum Mechanics, Prof. Mohammad Reza Pahlavani (Ed.), ISBN: 978-953-51-0058-4, InTech, Available from:

http://www.intechopen.com/books/measurements-in-quantum-mechanics/schr-dinger-transform-of-image-anew-tool-for-image-analysis

\section{INTECH}

open science | open minds

\section{InTech Europe}

University Campus STeP Ri Slavka Krautzeka 83/A

51000 Rijeka, Croatia Phone: +385 (51) 770447

Fax: +385 (51) 686166

www.intechopen.com

\section{InTech China}

Unit 405, Office Block, Hotel Equatorial Shanghai

No.65, Yan An Road (West), Shanghai, 200040, China

中国上海市延安西路65号上海国际贵都大饭店办公楼 405 单元

Phone: +86-21-62489820

Fax: +86-21-62489821 
(C) 2012 The Author(s). Licensee IntechOpen. This is an open access article distributed under the terms of the Creative Commons Attribution 3.0 License, which permits unrestricted use, distribution, and reproduction in any medium, provided the original work is properly cited. 